Casanoves Boix, J.; Küster Boluda, I. y Vila López, N. (2019). ¿Por qué las instituciones de educación superior deben apostar por la marca? Revista de Investigación Educativa, 37(1), 111-127.

DOI: http://dx.doi.org/10.6018/rie.37.1.291191

\title{
¿Por qué las instituciones de educación superior deben apostar por la marca?
}

\section{Why should higher education institutions invest in branding?}

\author{
Javier Casanoves Boix*, Inés Küster Boluda** y Natalia Vila López** \\ *Universidad Internacional de Valencia \\ ***niversidad de Valencia
}

\begin{abstract}
Resumen
A través de la presente investigación se pretende analizar el papel del capital de marca en el sector educativo. Para tal fin, se analizan las principales aportaciones de la literatura al estudio del capital de marca y su aplicación en el sector educativo, identificando qué variables determinan el mismo en la educación superior. Una vez establecido el modelo de capital de marca susceptible de aplicación en el sector educativo, se lleva a cabo un estudio empírico contando con una muestra cuantitativa de 2.239 respuestas válidas procedentes de distintos agentes universitarios implicados. Los resultados obtenidos muestran la repercusión del capital de marca en lo relativo a las variables que lo determinan, siendo: notoriedad de marca, imagen de marca, calidad percibida de marca y lealtad de marca.
\end{abstract}

Palabras clave: marketing; capital de marca; educación superior; agentes universitarios.

\begin{abstract}
This research was carried out to examine the role of brand capital in higher education. For this purpose, the main contributions of the literature related to the study of brand capital and its application in the educational sector were analyzed, identifying which variables determine brand capital in the higher education sector. Once the susceptible brand capital in the higher
\end{abstract}

Correspondencia: Javier Casanoves Boix, javier.casanoves@campusviu.es, Universidad Internacional de Valencia. 
education sector was established, an empirical study was carried out using a valid sample of 2,239 responses from different university agents involved. The results obtained show the repercussion of the brand capital relative to the determining variables, which are: brand awareness, brand image, perceived quality and brand loyalty.

Keywords: marketing; brand capital; higher education; university agents.

\section{Introducción}

En la actualidad, las universidades se encuentran operando dentro de entornos dinámicos y desafiantes, y la estrategia de marketing se ha convertido en una prioridad para asegurar un fuerte reclutamiento y retención de estudiantes y profesores (Asaad, Melewar, Cohen \& Balmer, 2013). Además, se busca obtener resultados en términos de valor, efectividad y beneficios potenciales (Küster, 2012). Por tanto, existe consenso en que la comprensión de la marca universitaria y el claro desarrollo y comunicación de la misma aportan gran valor para las instituciones educativas superiores (Duesterhaus \& Duesterhaus, 2014). Así pues, la gestión estratégica de la marca se convierte en pieza clave dentro del sector educativo superior (Rauschnabel, Krey, Babin \& Ivens, 2016).

Centrando nuestra atención en el papel que juega la educación superior en España, Angoita y Rahona (2007) consideran que el incremento del nivel educativo alcanzado en nuestro país en los últimos años ha sido uno de los fenómenos más relevantes acaecidos. Pese a que en algunos aspectos todavía no se han logrado la cota de otros países europeos, la trayectoria experimentada ha culminado en una convergencia educativa con los países de nuestro entorno. En este contexto, el crecimiento del sistema universitario español ha sido clave desde comienzos de la década de los noventa, representando un fenómeno sin precedentes ya que, hasta principios de los noventa, España se había caracterizado por presentar un déficit de capital humano en todos los niveles educativos (Angoita \& Rahona, 2007).

En este marco, los agentes universitarios implicados se convierten en pieza angular debido a que toda organización que ofrece un servicio (ya sea educativo o referido a cualquier otro sector) debe tener muy en cuenta la labor de sus empleados y clientes, lo cual es muy importante para construir marca poderosa (Lovelock, 1997). Todo construye o destruye marca, y dicho proceso continuo e indefinido en el tiempo debe alimentarse de todas las acciones que la empresa (o institución educativa) dirige a sus públicos, así como las interacciones y experiencias del público con la misma (Olins, 2008).

Así, y en el escenario descrito, la realización de la presente investigación supone un avance científico en la disciplina de marketing puesto que hasta la fecha, aunque se han realizado investigaciones específicas del capital de marca y las variables que lo componen (Buil, Martínez \& De Chernatony, 2010; Delgado \& Munuera, 2002; Faircloth, Capella \& Alford, 2001; Yoo \& Donthu, 2001), no existen investigaciones que analicen la percepción del capital de marca a través de opiniones de conjunto de los principales agentes implicados en instituciones educativas de carácter superior en España (Flavián \& Lozano, 2002). Asimismo, puede servir de ayuda a los gerentes y responsables universitarios puesto que pueden descifrar las claves en cuanto a las opiniones de sus empleados y clientes con respecto al capital de marca de la propia institución y generar, con ello, estrategias adecuadas para mantenerlo o mejorarlo. 


\section{La importancia de los agentes implicados en la educación superior}

Siguiendo a Webster, Hammond y Harmon (2006), el marketing es susceptible de aplicación a las universidades, ya sean públicas o privadas, ya que, ellas también tienen clientes, competencias e influencias externas, al tiempo que buscan lograr objetivos organizacionales. Su necesidad de supervivencia es similar a la utilidad en las empresas comerciales, específicamente, satisfacer a sus públicos en el largo plazo lo cual requiere que los ingresos sean adecuados para cubrir los gastos.

Ahora bien, en el ámbito docente puede resultar complicado definir quién es el cliente (Smith, 2003). Weaver (1976) identifica cuatro posibles clientes potenciales en el sistema educativo: el gobierno, sus administradores, los académicos y los consumidores actuales (los estudiantes, sus familias, empleados y sociedad en general). Robinson y Long (1987) distinguen entre consumidores primarios (los estudiantes), secundarios (los que pagan por ella como las autoridades educativas locales, empleadores, etc.) y terciarios (ex-estudiantes, padres, etc.). Flavián y Lozano (2001) consideran que el cliente prioritario es el alumno como receptor de la formación impartida en las instituciones educativas; las empresas, porque reciben un beneficio al contratar a los trabajadores con un determinado nivel de conocimiento y formación; la Administración Pública, porque asume los costes de esta actividad y que como consecuencia de ello espera obtener un rendimiento a cambio; y a la sociedad en su conjunto, por ser el máximo beneficiario de este tipo de servicios. Para Smith (2003), los clientes son todos: los estudiantes, el profesorado, los patrocinadores y los futuros empleadores.

De las propuestas de clientes planteados, la presente investigación centra su atención en aquellos agentes más directamente implicados: el estudiante (cliente externo), quien recibe los servicios del profesorado (cliente interno) y del personal de administración y servicios (cliente interno). Así, los tres agentes implicados poseen una importancia muy relevante dentro del sector educativo superior, ya sean profesores (Mas, 2011; Küster, 2012; Zabalza, 2009), empleados de servicios (Clotfelter, 2014; Douglas, McClelland \& Davies, 2008; Sultan \& Yin Wong, 2012) o estudiantes (Ibarra \& Michalus, 2014; Küster, 2012; Martín, García, Torbay \& Rodríguez, 2008).

A su vez, dichos agentes implicados cumplen una serie de funciones distintas que, sin duda, mejoran la notoriedad de marca educativa, la imagen que la misma proyecta, la calidad del servicio prestado y la lealtad por parte de los clientes. Así, tanto los profesores (Martínez García, García Domingo \& Quintanal Díaz, 2006; Küster, 2012; Casero, 2010), empleados de servicios (Mircea \& Andreescu, 2011; Thorsteinsson, Page \& Niculescu, 2010; Waterman, 2014) como estudiantes (Badía, 2007; Fernández de Morgado, 2015; Palazón-Pérez, Gómez-Gallego, Gómez-Gallego, Pérez-Cárceles \& Gómez García, 2011) son considerados capital humano clave de cara a generar capital de marca en las instituciones educativas superiores.

En suma, los tres agentes universitarios considerados tienen un papel fundamental dentro del sector educativo superior, colaborando en la vida académica y de la institución universitaria, al tiempo que se establecen mecanismos para que éstos expresen sus necesidades y deseos. Por tanto, deben ser considerados como activos clave de cara a transmitir y consumir los servicios educativos que las marcas universitarias ofrecen el mercado. 


\section{La percepción del capital de marca entre los agentes implicados}

Recordemos que el capital de marca puede ser definido como la relación individual y la experiencia de cada consumidor con la marca (Aaker, 1992; Wansink, 2003), aunque se pueden llegar a generalizar pautas y comportamientos universales en todos los consumidores (Farquhar, 1989).

Teniendo en cuenta dicha definición, numerosas han sido las aportaciones a la hora de delimitar los elementos que conforman dicho capital de marca. Tras la revisión de las siete propuestas más relevantes en la materia (Aaker, 1992; Buil et al., 2010; Delgado \& Munuera, 2002; Faircloth et al., 2001; Farquhar, 1989; Keller, 1993; Yoo \& Donthu, 2001), se han considerado cuatro elementos que son compartidos por los autores. Estos son: (1) notoriedad de marca, (2) imagen de marca, (3) calidad percibida y (4) lealtad de marca.

En relación a la investigación de cada uno de estos elementos en el contexto educativo, la Tabla 1 nos muestra las principales investigaciones. Como se comprueba, se pincelan percepciones del consumidor como piedra angular de cada uno de los elementos del capital de marca, los cuales tienen un papel fundamental en el presente estudio.

Tabla 1

Principales elementos del capital de marca en la educación superior.

\begin{tabular}{|c|c|c|c|c|c|c|c|c|}
\hline Componentes del capital marca & $\begin{array}{c}\text { Farquhar } \\
\text { (1999) }\end{array}$ & Aaker (1992) & Keller (1993) & $\begin{array}{l}\text { Faircloth, } \\
\text { Capellay } \\
\text { Alford (2001) }\end{array}$ & $\begin{array}{l}\text { Yoo y Donthu } \\
\text { (2001) }\end{array}$ & $\begin{array}{l}\text { Delgado y } \\
\text { Munuera } \\
\text { (2002) }\end{array}$ & $\begin{array}{c}\text { Buil, Martinez } \\
\text { y De } \\
\text { Chernatony } \\
\text { (2010) }\end{array}$ & Aportaciones del capital marca en la educación superior \\
\hline NOTORIEDAD DE MARCA & & $\mathbf{x}$ & $\mathbf{x}$ & & $\mathbf{x}$ & $x$ & $x$ & NOTORIEDAD DE MARCA EDUCATIVA \\
\hline Conciendia de marca & & & $\mathrm{x}$ & & $x$ & & & \multirow{5}{*}{$\begin{array}{c}\text { Koku, 1997; Morphew, 2001; Sevier, 2001; Toma, Dubrow y } \\
\text { Hartley, 2005; Brunzel, 2007; Brewer y Zhaa, 2010; Furey } \\
\text { Springer y Parsons, 2014 }\end{array}$} \\
\hline Familiaridad de marca & & $\bar{x}$ & $\mathrm{x}$ & & & & & \\
\hline Prominencia de marca & & & $\mathrm{x}$ & & & & & \\
\hline Reconodimiento marca & & & $\bar{x}$ & & & & & \\
\hline Recuerdo de marca & & & $\mathrm{x}$ & & & & & \\
\hline IMAGEN DE MARCA & $\mathbf{x}$ & $\mathbf{x}$ & $\mathbf{x}$ & $\mathbf{x}$ & $\mathbf{x}$ & $\mathbf{x}$ & $\mathbf{x}$ & IMAGEV DE MARCA EDUCATIVA \\
\hline Ascoiadiones de marca & $x$ & & $x$ & $x$ & $x$ & & $x$ & \multirow{7}{*}{ 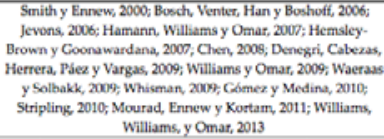 } \\
\hline Desempeño de marcáa & & & $\mathrm{x}$ & & & & & \\
\hline Diferenciación de la oferta & & $\bar{x}$ & & & & & & \\
\hline Imagineria de marca & & & $\bar{x}$ & & & & & \\
\hline Marca como organización & & $\mathrm{x}$ & & & & & & \\
\hline Persuralidad de marca & & $x$ & & & & & & \\
\hline Reputación de marca & & $x$ & & & & $x$ & & \\
\hline CALIDAD PERCIBIDA DE MARCA & & $\mathbf{x}$ & $x$ & & $\mathrm{x}$ & $\mathrm{x}$ & $x$ & CALIDAD PERCIBIDA DE MARCA EDUCATIVA \\
\hline Estima hacia la marca & & $\bar{x}$ & & & & & & \multirow{6}{*}{$\begin{array}{l}\text { Kissman y Van Tran, 1990; Ramsden, 1991; Byron, 1995; } \\
\text { Athiyaman, 1977; Vorthies, 1997; Booth, 1999; Binsardi y } \\
\text { Gwwulugo, 2003, Mai, 2005, Feltier, Schibrowsky y Drago, 2007, } \\
\text { Chen, 2008; Mourad, Ennew y Kortam, 2011; Pinar, Trapp, } \\
\text { Girard y Boyt, 2014 }\end{array}$} \\
\hline Juicios sobre la marca & & & $\bar{x}$ & & & & & \\
\hline Liderarga/Popularidad de marca & & $x$ & & & & & & \\
\hline Opinionus acurca de la marca & & & $\bar{x}$ & & & & & \\
\hline Sentimicntos hedia la marca & & & $x$ & & & & & \\
\hline Valor percibido & & $\mathrm{x}$ & & & & & & \\
\hline LEALTAD DE MARCA & $x$ & $x$ & $x$ & & $x$ & $x$ & $\mathrm{x}$ & LEALTAD DE MARCA EDUCATIVA \\
\hline AgradorSatisfacobón del cliente & & $x$ & & & & & & \multirow{6}{*}{$\begin{array}{l}\text { Nicholls, Harris, Morgan, Clarke y Sims, 1995; Nguyen y } \\
\text { LeBlanc, 2001; Lerman y Garbarino, 2002; Helgesen, 2008; Bok } \\
\text { 2009; Brown y Mazzarol, 2009; Pawsen y Ganesh, 2009; Rojas, } \\
\text { Vasquea, Kara y Cerda, 2009; Pinar, Trapp, Girard y Boyt, 2014 }\end{array}$} \\
\hline Confianza con la marca & & $x$ & & & & & & \\
\hline Evaluación pusitiva de marca & $x$ & & & & & & & \\
\hline Fidehidad de marca & & $x$ & & & & & & \\
\hline Repetióón de compra & & $x$ & & & & & & \\
\hline Resonancia de marca & & & $x$ & & & & & \\
\hline
\end{tabular}

En relación a la percepción del capital de marca entre los agentes universitarios implicados, Flavián y Lozano (2002) consideran que existe una corriente investigadora en nuestro país que señala la necesidad de una orientación al mercado por parte del profesor en instituciones universitarias. Por su parte, Brunner y Uribe (2007) explican que el nuevo escenario de la educación superior conlleva adecuar la enseñanza a un 
nuevo mercado universitario, en el que el rol del profesor es clave a la hora de generar imagen de marca educativa y, para ello, es necesario adecuar a los distintos agentes implicados en la institución educativa. Más aún, McAlexander, Koenig y Schouten (2006) explican que el personal de administración y servicios es considerado como un activo muy valioso para transmitir los servicios educativos que las marcas universitarias ofrecen al mercado. Y, Denegri, Etchebarne, Geldres, Cabezas y Herrera (2009) argumentan que las percepciones de los estudiantes influyen de manera significativa en la personalidad de marca, ya sea de universidad pública o privada.

En suma, y a la vista de lo argumentado en líneas previas, se considera de vital importancia tratar de descifrar las percepciones de dichos agentes implicados en su conjunto sobre el capital de marca educativo que exponemos en la presente investigación.

\section{HI. Notoriedad de marca}

La notoriedad de marca consiste en el desempeño del reconocimiento y recuerdo de la marca, caracterizada por la forma en que los consumidores adoptan sus decisiones (Aaker, 1992).

En el ámbito concreto de la educación, diversos autores (Brewer \& Zhao, 2010; Brunzel, 2007; Pinar, Furey, Springer \& Parsons, 2014) constatan en sus estudios que la notoriedad de marca en la educación superior es una variable muy importante para las universidades, de cara a generar mayor preferencia de consumo en servicios educativos y, con ello, incremento de ventas. Es decir, una mayor notoriedad de marca, supondrá un mayor capital de marca.

Por ejemplo, Brunzel (2007) argumenta que la nueva tendencia universitaria pasa por diseñar programas de marketing y branding basados en generar influencia positiva en los rankings universitarios y, con ello, mejorar la notoriedad de marca. Por su parte, Brewer y Zhao (2010) detallan que la notoriedad de marca de varias universidades de Sydney mejora cuando existe un gran conocimiento de marca, reputación, opiniones favorables de marca y calidad de la enseñanza. Y, Furey, Springer y Parsons (2014) explican que la notoriedad de marca de las universidades del Reino Unido se maximiza cuando la marca educativa ofrece un claro posicionamiento, experiencia, aspiración de estudio y colaboración con el medio ambiente.

A la vista de lo expuesto en líneas previas, es posible plantear la primera hipótesis de investigación, H1: La percepción sobre notoriedad de marca influye en la percepción del capital de marca en las instituciones de educación superior.

\section{H2. Imagen de marca}

Las imágenes mentales que se pueda hacer un consumidor de una marca están íntegramente relacionadas con el desempeño y el esfuerzo que ésta realice en su creación de valor, los cuales a su vez se relacionan con las asociaciones, diferenciación de producto e imagen y personalidad que transmite la marca (Keller, 1993).

En el ámbito concreto de la educación, diversos autores (Chen, 2008; Denegri et al., 2009; Hernández \& Zamora, 2010; Hamann, Williams \& Omar, 2007; Waeraas \& Solbakk, 2009; Whisman, 2009; Williams \& Omar, 2009; Williams, Williams \& Omar, 2013) constatan que la imagen de marca en las universidades públicas y privadas es un atractivo muy valioso para las organizaciones, debiéndose realizar mediciones 
eficaces y rápidas de la imagen trasmitida por los públicos objetivos puesto que dicha percepción puede resultar diferente en ambos ámbitos. Esta imagen, en la medida que mejore, conllevará aumentos del capital de marca de la institución universitaria cuya imagen se vea favorecida.

Por ejemplo, Hamann et al. (2007) detallan en su estudio que el prestigio de la institución educativa está por encima de las necesidades que ésta pueda satisfacer a la hora de maximizar la imagen de marca educativa. Por su parte, Hernández y Zamora (2010) explican que las universidades españolas deben generar políticas de marketing y estrategias de comunicación eficaces para mejorar la imagen de marca de la institución. Y, Carmelo y Calvo (2010) creen que las universidades españolas tienen el reto de romper posiciones perezosas que se resisten a creer que el estudiante puede ser considerado como un cliente, aplicando técnicas de gestión de imagen que van más allá de estrategias y tácticas de marketing de un producto o servicio concreto.

A la vista de lo expuesto en líneas previas, es posible plantear la segunda hipótesis de investigación, H2: La percepción sobre imagen de marca influye en la percepción del capital de marca en las instituciones de educación superior.

\section{H3. Calidad percibida de marca}

La calidad percibida se basa en las actitudes hacia las marcas, siendo evaluaciones generales que forman parte de la elección de consumo por parte de los consumidores. $\mathrm{Y}$ estas actitudes dependen, generalmente, de los atributos y beneficios específicos de la marca (Wilkie, 1994).

En el ámbito concreto de la educación, diversas investigaciones (Binsardi \& Ekwulugo, 2003; Chen, 2008; Mourad, Ennew \& Kortam, 2011; Peltier, Schibrowsky \& Drago, 2007; Pinar et al., 2014) constatan que la calidad percibida en la educación superior, privada y pública, es una variable a tener muy en cuenta también, ya que de ella depende que las instituciones educativas obtengan mayor satisfacción por parte de sus usuarios. La calidad, por tanto, es otra fuente clave en la configuración del capital de la marca de la institución universitaria, en tanto en cuanto mayor calidad percibida, se traducirá en mayor capital de marca.

Por ejemplo, Binsardi y Ekwulugo (2003) explican que las universidades del Reino Unido, en aras de seguir incrementando el número de matrículas año a año, deben mejorar la calidad percibida que tienen los clientes potenciales sobre la institución, diseñando estrategias de fijación de precios, promociones y comercialización de su tipo de educación en el extranjero. Por su parte, Chen (2008) argumenta que la calidad percibida hacia las universidades canadienses genera percepción positiva a nivel internacional y, con ello, deseo de adquisición de servicio educativo. Y, Pinar et al. (2014) demuestran que la calidad percibida es la variable más importante a tener en cuenta en la construcción de marcas universitarias poderosas, seguida de la reputación de marca y el ambiente emocional. Con ello, el servicio de biblioteca es el servicio más determinante, seguido de la residencia del estudiante (el hall de la entrada y la cafetería), el desarrollo profesional y las instalaciones (ej. gimnasio, clase, laboratorios, etc.)

A la vista de lo expuesto en líneas previas, es posible plantear la tercera hipótesis de investigación, H3: La percepción sobre calidad percibida de marca influye en la percepción del capital de marca en las instituciones de educación superior. 


\section{H4. Lealtad de marca}

La lealtad de marca se asocia con el comportamiento del cliente hacia su repetición de compra, traduciendo dicha lealtad como la disposición a pagar mayores precios por la misma, menores costes de servicio y el incremento de nuevos clientes a través de la atracción (Aaker, 1996).

En el ámbito concreto de la educación, trabajos diversos (Bok, 2003; Brown \& Mazzarol, 2009; Nguyen \& LeBlanc, 2001; Pinar et al., 2014; Rojas-Méndez, VasquezParraga, Kara \& Cerda-Urrutia, 2009) constatan la especial relevancia que tiene la lealtad de marca en el sector educativo superior, lo que implica que dicha variable es muy importante a la hora de generar capital de marca educativo. Públicos más leales contribuyen a incrementar el capital de marca.

Por ejemplo, Nguyen y LeBlanc (2001) exponen que el grado de fidelidad aumenta cuando las percepciones sobre la reputación e imagen de marca institucional son favorables. Además, la interacción con el estudiante ayuda a explicar con mayor fidelidad de los clientes. Por su parte, Bok, 2009 considera que las universidades han incluido en su plan anual de enseñanza una estrategia de comercialización de los servicios ofrecidos, siendo la lealtad de marca la variable más importante a la hora de generar venta cruzada entre productos educativos. Brown y Mazzarol (2009) explican que los estudiantes australianos tienen mayor lealtad hacia su universidad a medida que se le satisfacen sus necesidades. Así, se convierte en una variable a tener muy en cuenta, sobre todo, por universidades de menor prestigio que buscan competir en un mercado cada vez más desregulado y basado en un ambiente de mercado empresarial.

A la vista de lo expuesto en líneas previas, es posible plantear la cuarta hipótesis de investigación, H4: La percepción sobre lealtad de marca influye en la percepción del capital de marca en las instituciones de educación superior.

Con todo ello, la Figura 1 muestra nuestra propuesta de modelo teórico diseñado para defender la presente investigación.

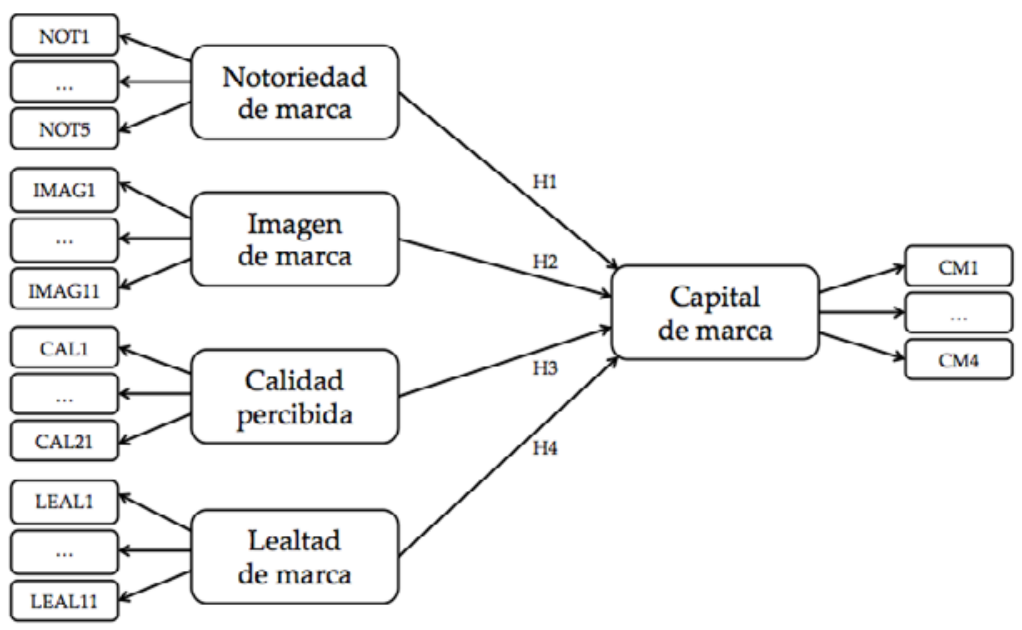

Figura 1. Modelo teórico propuesto para la presente investigación. 


\section{Método}

\section{Objetivos}

Los objetivos del presente artículo se centran en: (1) analizar qué se entiende por capital de marca y cuáles son sus variables determinantes en el sector educativo, y (2) descifrar a nivel empírico cuáles son las variables más destacables del capital de marca educativo por parte de los agentes implicados.

\section{Población y Muestra}

Para cuantificar la población objetivo se utilizaron datos oficiales de Facultades relacionadas con el ámbito de Economía de ocho instituciones educativas superiores de Valencia (España), y en el que se trabajó con un total de 1.304 agentes de universidad pública y 1.038 de universidad privada. Tras la recogida y depuración de la información, se obtuvieron un total de 2.239 respuestas válidas.

Como datos destacables del perfil de la muestra, se trata de un grupo mixto de hombres y mujeres (muestra muy heterogénea) procedentes de universidad pública y privada, de 33 años de edad media y con respuestas procedentes de 36 nacionalidades distintas, siendo España el país que mayor número representa (88\%). Con ello, cerca del $51 \%$ de la muestra procede de estudiantes universitarios, el 30\% de profesores y el $19 \%$ de personal de administración y servicios. Además, y en lo relativo a antigüedad, cerca del $58 \%$ de profesores y el 54\% de respuestas del personal de servicios se sitúan en torno a más de 10 años y con contrato laboral a tiempo completo. Así, se considera que las respuestas recibidas por la mayoría de encuestados son de calidad, ya que provienen de agentes universitarios con buenos conocimientos del funcionamiento y el saber hacer de su universidad. Y, destacar la gran participación obtenida y el bajo porcentaje de encuestados que han omitido responder a alguna de las preguntas de clasificación (5.76\%).

\section{Instrumento}

La recogida de información se realizó a través de un cuestionario en formato digital y papel. Se empleó un muestreo no probabilístico por conveniencia. Además, se emplearon escalas Likert de grado 5 para medir los conceptos de (1) notoriedad de marca, (2) imagen de marca, (3) calidad percibida de marca y (4) lealtad de marca. Dichas escalas están basadas en las escalas de medición propuestas por Aaker (1992) y Keller (1993), y se han adaptado los correspondientes ítems a nuestro ámbito de estudio, la educación superior.

\section{Procedimiento de recogida y análisis de datos}

Las técnicas para el análisis de datos están basadas en la estadística descriptiva y el análisis multivariante, recurriéndose como herramienta de trabajo al SPSS v.19 para Windows para las técnicas descriptivas de los datos y al EQS 6.2 para ejecutar las técnicas multivariantes. El procesamiento estadístico de los datos seguido en 
este estudio supone la aplicación de distintos métodos de análisis en función de la información que se desea obtener, distinguiendo entre: (1) descripción y clasificación de los datos, y (2) contraste de hipótesis.

\section{Resultados}

Previo al contraste de hipótesis, se analizaron las características psicométricas del instrumento de medida. Para ello, se dividió la información en (1) un análisis de la calidad de los ítems y (2) la validación de las escalas, la cual se muestra en la Tabla 2.

Tabla 2

Fiabilidad y validez convergente

\begin{tabular}{|c|c|c|c|c|c|c|c|}
\hline Factor & Indicador & Carga & $t$ Robusto & $\bar{X}$ Carga & CA & CR & AVE \\
\hline & NOT1 & $0.37^{* * *}$ & 13.96 & & & & \\
\hline NOT. & NOT2 & $0.81^{* * *}$ & 33.69 & & & & \\
\hline \multirow{7}{*}{$\begin{array}{l}\text { Notoriedad } \\
\text { de marca }\end{array}$} & NOT3 & $0.87^{* * *}$ & 40.88 & 0.71 & 0.84 & 0.85 & 0.54 \\
\hline & NOT4 & $0.82^{* * *}$ & 38.95 & & & & \\
\hline & NOT5 & $0.70^{* * *}$ & 28.20 & & & & \\
\hline & IMAG1 & $0.67^{* * *}$ & 28.26 & & & & \\
\hline & IMAG2 & $0.79^{* * *}$ & 32.87 & & & & \\
\hline & IMAG3 & $0.81^{* * *}$ & 37.83 & & & & \\
\hline & IMAG4 & $0.71^{* * *}$ & 31.87 & & & & \\
\hline \multirow{15}{*}{$\begin{array}{l}\text { IMAG. } \\
\text { Imagen de } \\
\text { marca }\end{array}$} & IMAG5 & $0.46^{* * *}$ & 17.15 & & & & \\
\hline & IMAG6 & $0.75^{* * *}$ & 33.86 & 0.65 & 0.90 & 0.89 & 0.44 \\
\hline & IMAG7 & $0.68^{* * *}$ & 30.26 & & & & \\
\hline & IMAG8 & $0.36^{* * *}$ & 13.11 & & & & \\
\hline & IMAG9 & $0.69^{* * *}$ & 28.63 & & & & \\
\hline & IMAG10 & $0.68^{* * *}$ & 29.45 & & & & \\
\hline & IMAG11 & $0.70^{* * *}$ & 30.41 & & & & \\
\hline & CAL1 & $0.73^{* * *}$ & 33.06 & & & & \\
\hline & CAL2 & $0.55^{* * *}$ & 22.01 & & & & \\
\hline & CAL3 & $0.75^{* * *}$ & 35.70 & & & & \\
\hline & CAL4 & $0.78^{* * *}$ & 36.84 & & & & \\
\hline & CAL5 & $0.64^{* * *}$ & 27.09 & & & & \\
\hline & CAL6 & $0.62^{* * *}$ & 25.97 & & & & \\
\hline & CAL7 & $0.61^{* * *}$ & 24.29 & & & & \\
\hline & CAL8 & $0.59^{* * *}$ & 23.68 & & & & \\
\hline CAL. & CAL9 & $0.64^{* * *}$ & 27.69 & & & & \\
\hline Calidad & CAL10 & $0.60^{* * *}$ & 26.21 & & & & \\
\hline percibida de & CAL11 & $0.60^{* * *}$ & 25.05 & 0.67 & 0.95 & 0.95 & 0.46 \\
\hline marca & CAL12 & $0.62^{* * *}$ & 25.38 & & & & \\
\hline
\end{tabular}




\begin{tabular}{|c|c|c|c|c|c|c|c|}
\hline & CAL13 & $0.80^{* * *}$ & 38.86 & & & & \\
\hline & CAL14 & $0.72^{* * *}$ & 32.85 & & & & \\
\hline & CAL15 & $0.70^{* * *}$ & 31.14 & & & & \\
\hline & CAL16 & $0.61^{* * *}$ & 24.84 & & & & \\
\hline & CAL17 & $0.71^{* * *}$ & 32.43 & & & & \\
\hline & CAL18 & $0.75^{* * *}$ & 36.13 & & & & \\
\hline & CAL19 & $0.70^{* * *}$ & 32.60 & & & & \\
\hline & CAL20 & $0.75^{* * *}$ & 33.42 & & & & \\
\hline & CAL21 & $0.66^{* * *}$ & 26.39 & & & & \\
\hline & LEAL1 & $0.79^{* * *}$ & 37.50 & & & & \\
\hline & LEAL2 & $0.83^{* * *}$ & 41.24 & & & & \\
\hline & LEAL3 & $0.80^{* * *}$ & 39.22 & & & & \\
\hline & LEAL4 & $0.84^{* * *}$ & 44.05 & & & & \\
\hline LEAL. & LEAL5 & $0.81^{* * *}$ & 41.24 & & & & \\
\hline Lealtad de & LEAL6 & $0.57^{* * *}$ & 21.99 & 0.74 & 0.94 & 0.93 & 0.55 \\
\hline marca & LEAL7 & $0.80^{* * *}$ & 38.34 & & & & \\
\hline & LEAL8 & $0.52^{* * *}$ & 19.61 & & & & \\
\hline & LEAL9 & $0.78^{* * *}$ & 35.98 & & & & \\
\hline & LEAL10 & $0.72^{* * *}$ & 33.42 & & & & \\
\hline & LEAL11 & $0.63^{* * *}$ & 29.12 & & & & \\
\hline & CM1 & $0.53^{* * *}$ & 21.04 & & & & \\
\hline CM. Capital & CM2 & $0.70^{* * *}$ & 31.31 & 0.68 & 0.79 & 0.78 & 0.47 \\
\hline de marca & CM3 & $0.82^{* * *}$ & 38.29 & & & & \\
\hline & CM4 & $0.67^{* * *}$ & 27.62 & & & & \\
\hline
\end{tabular}

Nota: $\mathrm{N}=2.239 ;{ }^{* * *} \mathrm{p}<0.01 ;{ }^{* *} \mathrm{p}<0.05 ;{ }^{*} \mathrm{p}<0.1$; Satorra-Bentler $\mathrm{X}^{2}(\mathrm{p})=9.377,016(0.0000), \mathrm{df}=1.263$ $\mathrm{CFI}=0.81 ; \mathrm{NFI}=0.80 ; \mathrm{NNFI}=0.80 ; \mathrm{IFI}=0.81 ; \mathrm{RMSEA}=0.067$

La fiabilidad se comprobó mediante tres métodos de análisis, siendo: (1) a de Cronbach (CA) obteniendo, en todos los casos, valores superiores a 0.7 (Nunnally \& Bernstein, 1994); (2) análisis de fiabilidad compuesta (CR) obteniendo también valores superiores 0.7 (Carmines \& Zeller, 1979); y (3) análisis de la varianza extraída promedio (AVE), donde los resultados fueron cercanos, iguales o superiores a 0.5 (Fornell \& Larcker, 1981).

Además, las medidas de bondad de ajuste de los modelos presentan valores aceptables $(\mathrm{NFI}=0.80 ; \mathrm{NNFI}=0.80 ; \mathrm{CFI}=0.81 ; \mathrm{IFI}=0.81$ ) ya que están cerca de 0.9 (Hair, Black, Babin, Anderson \& Tatham, 2005) y el indicador RMSEA = 0.067 también presenta un buen ajuste, siendo su valor inferior a 0.08 (Steiger, 1990), sugiriendo que el modelo estructural encaja bien con la estructura de datos. Y, aunque no todos los valores de las cargas factoriales por separado son superiores a 0.6 (Bagozzi \& Yi, 1988), bien es cierto que los a de Cronbach de cada uno de los factores son superiores a 0.70, (Nunnally \& Bernstein, 1994), los valores del análisis de fiabilidad compuesta (CR) son superiores 0.70 (Carmines \& Zeller, 1979) 
y el promedio de las cargas factoriales estandarizadas () son cercanos o superiores a 0.7 (Currás, 2007), constatándose así la validez convergente del modelo propuesto.

Finalmente, y mediante un modelo de ecuaciones estructurales basado en el método de máxima verosimilitud robusto, se llevó a cabo el contraste de hipótesis, tal y como se muestra en la Tabla 3.

Como se puede observar, los resultados sugieren que el modelo diseñado en esta investigación aplicado al conjunto de agentes implicados resulta satisfactorio para explicar 3 de las 4 hipótesis planteadas. Así, se demostró una relación positiva entre las percepciones de notoriedad de marca, imagen de marca y lealtad de marca con respecto al capital de marca, aceptando la hipótesis primera $(\mathrm{H} 1: \beta=0.215 ; \mathrm{p}<0.01)$, la hipótesis segunda $(\mathrm{H} 2: \beta=0.558 ; \mathrm{p}<0.01)$ y la hipótesis cuarta $(\mathrm{H} 4: \beta=0.207$; $\mathrm{p}<$ $0.01)$. Es decir, se irá consolidando una percepción favorable hacia el capital de marca a medida que se vayan construyendo percepciones positivas hacia cada una de las tres variables revisadas en la presente investigación. Sin embargo, y en lo relativo a la hipótesis tercera (H3: $\beta=0.069 ; \mathrm{ns})$, cabe destacar que no se demostró una relación positiva entre la percepción de calidad percibida de marca y el capital de marca por parte de los 2.239 agentes universitarios encuestados.

Tabla 3

Contraste de hipótesis

\begin{tabular}{clccc}
\hline Hipótesis & \multicolumn{1}{c}{ Relación Estructural } & $\beta$ Estand. & t Robusto & Criterio \\
\hline H1 & $\begin{array}{l}\text { Percepción Notoriedad de Marca --> } \\
\text { Percepción Capital de Marca }\end{array}$ & $0.215^{* * *}$ & 6.00 & Aceptada \\
H2 & $\begin{array}{l}\text { Percepción Imagen de Marca --> } \\
\text { Percepción Capital de Marca }\end{array}$ & $0.558^{* * *}$ & 7.59 & Aceptada \\
H3 & $\begin{array}{l}\text { Percepción Calidad Percibida --> } \\
\text { Percepción Capital de Marca }\end{array}$ & 0.069 & 1.02 & Rechazada \\
H4 & $\begin{array}{l}\text { Percepción Lealtad de Marca --> } \\
\text { Percepción Capital de Marca }\end{array}$ & $0.207^{* * *}$ & 4.92 & Aceptada \\
\hline
\end{tabular}

Nota: $\mathrm{N}=2.239 ;{ }^{* * *} \mathrm{p}<0.01 ;{ }^{* *} \mathrm{p}<0.05 ;{ }^{*} \mathrm{p}<0.1$; Satorra-Bentler $\mathrm{X}^{2}(\mathrm{p})=9.377,016(0.0000), \mathrm{df}=1.263$

$\mathrm{CFI}=0.81 ; \mathrm{NFI}=0.80 ; \mathrm{NNFI}=0.80 ; \mathrm{IFI}=0.81 ; \mathrm{RMSEA}=0.067$

\section{Discusión y conclusiones}

Como conclusiones principales relacionadas con la educación superior, cabe destacar que se sintetizan elementos comunes a través de la revisión literaria (Alesón, 1986; Becker, 1964; Bok, 2003; Brunner \& De Gobierno, 2005; Gibbons, Limoges, Nowotny, Schawartzman, Scott \& Trow, 1994; Marcenario \& Navarro, 2001) para aproximar en líneas generales el concepto y sus características principales, destacando que: (1) los individuos demandan educación con el objetivo de mejorar su productividad laboral, (2) sin la percepción de un beneficio compensatorio futuro, y desde una perspectiva económica, las acciones educativas no podrían justificarse, ya que son muy costosas, (3) 
las instituciones educativas superiores se dividen en tres categorías de desarrollo: elitistas, masivas y universales, (4) la explosión de la información académica y del conocimiento avanzado está llevando a cabo cambios de curso que buscan garantizar oportunidades de formación permanente a lo largo de la vida de las personas, alterando estructuras y orientaciones curriculares de tal modo que se proporcione una educación general básica y competencias profesionales sintonizadas con los requerimientos del mercado laboral, y (5) las instituciones educativas superiores responden a las mayores demandas por acceso mediante un incremento de la oferta y una diversificación de oportunidades, lo que lleva a una creciente diferenciación y mayor complejidad de los sistemas.

Como conclusiones generales de los resultados obtenidos en el contraste de hipótesis, destacar que el modelo global planteado demostró una relación positiva y directa en las percepciones de notoriedad de marca (Furey et al., 2014; Mourad et al., 2011), imagen de marca (Cervera, Schlesinger, Mesta \& Sánchez, 2012; Cubillo, Sánchez \& Cerviño, 2006; Webb \& Jagun, 1997), lealtad de marca (Cervera et al., 2012; Pinar et al., 2014; Rojas-Méndez et al., 2009; Webb \& Jagun, 1997) y capital de marca. Sin embargo, no se demostró un vínculo afirmativo entre la percepción de calidad percibida de marca y capital de marca por parte de los 2.239 agentes universitarios estudiados.

Fruto de los resultados y conclusiones alcanzadas es posible plantear cuatro implicaciones gerenciales. En primer lugar, y con respecto a la notoriedad de marca, los resultados muestran que las instituciones educativas superiores están realizando un gran trabajo de posicionamiento de marca, planteando acciones de marketing dirigidas a sus agentes universitarios. Tales acciones han conseguido generar gran boca oído entre su capital humano, alto conocimiento y buenas opiniones sobre el centro e interesantes recomendaciones en caso de decidir realizar unos estudios de grado o postgrado. Aun así, convendría incrementar su participación en redes sociales, ya que se considera que puede mejorar también su conocimiento de marca entre los mismos, los cuales están cada vez más conectados a la Red pueden ser tentados con información de interés de otras marcas educativas.

En segundo lugar, y en lo relativo a la imagen de marca, destacar que es la variable más valorada por el conjunto de agentes implicados. Así, éstos tienen buena opinión en cuanto a precio-calidad, transmitiendo la propia universidad confianza, admiración y crecimiento profesional a sus trabajadores y estudiantes. Además, muchos de ellos consideran que otras personas a las que admiran les gustaría trabajar o estudiar en sus centros, lo que nos hace pensar que han conseguido plasmar correctamente su buen hacer entre su público. Con ello, los agentes implicados satisfechos actúan como brand ambassadors de sus instituciones educativas, lo cual hace que los gerentes deban contemplar, en sus programas de marketing, estrategias adecuadas para premiar dicha implicación, así como desarrollar estrategias que encaucen las asociaciones que tienen sus propios trabajadores y estudiantes con la marca educativa.

En tercer lugar, y en cuanto a calidad percibida de marca, destacar que existe falta de consenso en cuanto a satisfacción de las necesidades. Así, y con respecto al equipo docente y de administración, bien es cierto que se ha se ha conseguido crear un buen equipo de trabajo, con relaciones interprofesionales sólidas y estables entre grupos de trabajo, consiguiendo además que éstos se preocupen por los alumnos y por sus compañeros de trabajo, pero reina la preocupación que tienen ciertos profesionales 
por la falta de estabilidad laboral y la escasa contraprestación económica recibida, lo que hace que la calidad percibida sea un elemento que puntúa negativamente en el capital de marca educativo. Por tanto, se considera que los gerentes deben mejorar sus servicios e instalaciones $y$, a posteriori, incentivar a capital interno con programas de formación y mejora salarial, para que éstos desarrollen con más pasión su trabajo, sean más innovadores y el resultado pueda percibirse por parte de los estudiantes.

En cuarto y último lugar, y en lo relativo a lealtad de marca, cabe destacar que, aunque la mayoría de los encuestados están orgullosos de que los demás sepan que trabajan o estudian en su centro universitario, muchos de ellos no se identifican con los valores de las propias instituciones, teniendo un bajo sentido de pertenencia a las mismas y apenas simpatizando con sus compañeros de trabajo a nivel de valores humanos y organizativos. Ello nos hace pensar que los gerentes educativos deben incrementar su apuesta por actividades que generen espíritu de equipo, buscando maximizar las relaciones personales entre los agentes universitarios implicados y mejorando, con ello, la fidelidad hacia la propia marca.

Por su parte, y hablando de limitaciones de la investigación, destacar que existe un quinto elemento (otros activos de la marca, o también llamado comportamiento del mercado) susceptible de ser incorporado como variable del capital de marca; elemento que ha sido defendido por diversos autores (Aaker, 1992; Delgado \& Munuera, 2002; Faircloth et al., 2001; Farquhar, 1989). Otra cuestión a considerar es la referida al tamaño de la muestra, considerando que, aunque se han estudiado ocho instituciones educativas completamente heterogéneas, si se hubiera ampliado la misma a más instituciones educativas superiores (públicas y privadas) habrían aumentado la calidad de la presente investigación. Además, se ha realizado una técnica íntegramente cuantitativa, creyendo que si se hubiera complementado con un estudio cualitativo posiblemente habría mejorado también dicha calidad.

Finalmente, y hablando de futuras líneas de investigación, resultaría especialmente interesante complementar la presente investigación con un estudio de carácter digital, de cara a generar análisis comparativos entre el entorno offline y online y comprobar, con ello, si efectivamente las percepciones que tienen los distintos agentes universitarios estudiados sería la misma en ambos ecosistemas.

\section{Referencias}

Aaker, D. A. (1992). The value of brand equity. Journal of business strategy, 13(4), 27-32. Aaker, D. A. (1996). Building Strong Brands. Nueva York: The Free Press.

Alesón, C. L. (1986). Enseñanza pública y privada en España: sobre el porvenir de una ilusión. En M. Fernández Enguita (Coord.) Marxismo y Sociología de la Educación (pp. 331-372). Madrid: Akal.

Angoita, M., \& Rahona, M. (2007). Evolución de la educación universitaria en España: diferentes perspectivas y principales tendencias (1991-2005). Revista de educación, 344, 219-221.

Asaad, Y., Melewar, T. C., Cohen, G., \& Balmer, J. (2013). Universities and export market orientation: An exploratory study of UK post-92 universities. Marketing Intelligence $\mathcal{E}$ Planning, 31(7), 838-856. doi: https://doi.org/10.1108/MIP-01-2013-0007 
Badía, T. (2007). Ayuda al aprendizaje con tecnología en la educación superior. RUSC. Universities and Knowledge Society Journal, 3(2), 5-19.

Bagozzi, R. P., \& Yi, Y. (1988). On the evaluation of structural equation models. Journal of the academy of marketing science, 16(1), 74-94.

Becker, G. S. (1964). Human capital: a theoretical analysis with special reference to education. Chicago: University of Chicago Press.

Binsardi, A., \& Ekwulugo, F. (2003). International marketing of British education: research on the students' perception and the UK market penetration. Marketing Intelligence E Planning, 21(5), 318-27. doi: https://doi.org/10.1108/02634500310490265

Bok, D. (2003). Universities in the Marketplace: The Commercialization of Higher Education. Princeton: Princeton University Press.

Brewer, A., \& Zhao, J. (2010). The impact of a pathway college on reputation and brand awareness for its affiliated university in Sydney. International Journal of Educational Management, 24(1), 34-47. doi: https://doi.org/10.1108/09513541011013033

Brown, R. M., \& Mazzarol, T. W. (2009). The importance of institutional image to student satisfaction and loyalty within higher education. Higher Education, 58(1), 81-95. doi: https://doi.org/10.1007/s10734-008-9183-8

Brunner, J. J., \& De Gobierno, E. (2005). Tendencias recientes de la educación superior a nivel internacional: marco para la discusión sobre procesos de aseguramiento de la calidad. (Documento de trabajo). Viña del Mar: Universidad Adolfo Ibáñez.

Brunner, J. J., \& Uribe, D. (2007). Mercados universitarios: el nuevo escenario de la educación superior. Santiago de Chile: Ediciones Universidad Diego Portales.

Brunzel, D. L. (2007). Universities sell their brands. Journal of Product E Brand Management, 16(2), 152-153. doi: https://doi.org/10.1108/10610420710740034

Buil, I., Martínez, E., \& De Chernatony, L. (2010). Medición del valor de marca desde un enfoque formativo. Cuadernos de Gestión, 10(nº especial), 167-196. doi: https:// doi.org/10.5295/cdg.100204ib

Carmelo, M., \& Calvo, S. (2010). Branding universitario: marcando la diferencia. Madrid: Delta.

Carmines, E. G., \& Zeller, R. A. (1979). Reliability and validity assessment. Londres: Sage publications.

Casero, A. (2010). ¿Cómo es el buen profesor universitario según el alumnado?. Revista española de pedagogía, 68(246), 223-242.

Cervera, A., Schlesinger, W., Mesta, M. Á., \& Sánchez, R. (2012). Medición de la imagen de la universidad y sus efectos sobre la identificación y lealtad del egresado: una aproximación desde el modelo de Beerli y Díaz (2003). Revista Española de Investigación en Marketing ESIC, 16(2), 7-29.

Chen, L. H. (2008). Internationalization or international marketing? Two frameworks for understanding international students' choice of Canadian universities. Journal of Marketing for Higher Education, 18(1), 1-33. Doi: https://doi.org/10.1080/08841240802100113

Clotfelter, C. T. (2014). Buying the best: Cost escalation in elite higher education. Princeton: Princeton University Press.

Cubillo, J., Sánchez, J., \& Cervino, J. (2006). International students' decision-making process. International Journal of Educational Management, 20(2), 101-115. doi: https:// doi.org/10.1108/09513540610646091 
Currás, R. (2007). Comunicación de la responsabilidad social corporativa: imagen e identificación con la empresa como antecedentes del comportamiento del consumidor. (Tesis de doctorado). Recuperada de RODERIC (http://roderic.uv.es/handle/10550/38448)

Delgado, E. \& Munuera, J. L. (2002). Medición del capital de marca con indicadores formativos. Investigación y Markerting, 759, 16-20.

Denegri, M., Etchebarne, M. S., Geldres, V., Cabezas, D., \& Herrera, V. (noviembre, 2009). Personalidad de marca de las carreras de ciencias empresariales: un análisis corporativo entre universidad pública y privada. Trabajo presentado en la XLIV Asamblea Anual del Consejo Latinoamericano de Escuelas de Administración (CLADEA), Guayaquil.

Douglas, J., McClelland, R., \& Davies, J. (2008). The development of a conceptual model of student satisfaction with their experience in higher education. Quality Assurance in Education, 16(1), 19-35. doi: https://doi.org/10.1108/09684880810848396

Duesterhaus, A. P., \& Duesterhaus, M. (2014). Attributes of successful university brands in the U.S.A. Journal of Brand Strategy, 3(2), 169-183.

Farquhar, P. H. (1989). Managing brand equity. Marketing research, 1(3), 24-33.

Faircloth, J. B., Capella, L. M., \& Alford, B. L. (2001). The effect of brand attitude and brand image on brand equity. Journal of Marketing Theory and Practice, 9(3), 61-75. doi: https://doi.org/10.1080/10696679.2001.11501897

Fernández de Morgado, N. (2015). Retención y Persistencia Estudiantil en Instituciones de Educación Superior: Una revisión de la literatura. Paradigma, 30(2), 39-61.

Flavián, C., \& Lozano, F. J. (2002). Análisis de la relación entre la actitud y el comportamiento orientado al mercado en la Universidad Pública española. En T. Luque (Coord.) XIV Encuentro de Profesores Universitarios de Marketing (pp. 531-546). Madrid: ESIC editorial.

Fornell, C., \& Larcker, D. F. (1981). Structural equation models with unobservable variables and measurement error: Algebra and statistics. Journal of marketing research, 18(3), 382-388. doi: http://dx.doi.org/10.2307/3150980

Furey, S., Springer, P., \& Parsons, C. (2014). Positioning university as a brand: distinctions between the brand promise of Russell Group, 1994 Group, University Alliance, and Million+ universities. Journal of Marketing for Higher Education, 24(1), 99-121. doi: https://doi.org/10.1080/08841241.2014.919980

Gibbons, M., Limoges, C., Nowotny, H., Schwartzman, S., Scott, P., \& Trow, M. (1994). The new production of knowledge: The dynamics of science and research in contemporary societies. Londres: Sage Publications.

Hair, J. F., Black, W. C., Babin, B. J., Anderson, R. E., \& Tatham, R. L. (2005). Multivariate data analysis. (Vol. 7.) Upper Saddle River, Nueva Jersey: Prentice Hall.

Hamann, D., Williams, R., \& Omar, M. (2007). Branding Strategy and Consumer HighTechnology Product. The Journal of Product \& Brand Management, 16(2), 98-111.

Hernández, F., \& Zamora, R. (2010). Diagnóstico de la imagen de marca de las instituciones universitarias en España. En J. M. Pestano, S. Toledano, A. I. Ardévol \& C. E. Hernández (Coords.) Actas II Congreso Internacional Latina de Comunicación Social. La Comunicación Social, en estado crítico: entre el mercado y la comunicación para la libertad (pp. 1-20). Tenerife: Sociedad latina de comunicación social.

Ibarra, C. M., \& Michalus, J. C. (2014). Análisis del rendimiento académico mediante un modelo Logit. Revista Ingeniería Industrial, 9(2), 47-56. 
Instituto Valenciano de Estadística (2008). La contribución socioeconómica de las universidades valencianas. Valencia: Autor.

Keller, K. L. (1993). Conceptualizing, measuring, and managing customer-based Brand equity. Journal of Marketing, 57, 1-22.

Küster, I. (2012). El Docente Universitario desde una perspectiva de mercado: Influencia en el rendimiento del estudiante. Alicante: 3 ciencias.

Lovelock, C. H. (1997). Mercadotecnia de servicios. México: Prentice-Hall Hispanoamericana.

Marcenario, O. D., \& Navarro, M. L. (2001). Un análisis microeconómico de la demanda de educación superior en España. Estudios de economía aplicada, 19, 69-86.

Martín, E., García, L. A., Torbay, A., \& Rodríguez, T. (2008). Estrategias de aprendizaje y rendimiento académico en estudiantes universitarios. International Journal of Psychology and Psychological Therapy, 8(3), 401-412.

Martínez García, M. M., García Domingo, B., \& Quintanal Díaz, J. (2006). El perfil del profesor universitario de calidad desde la perspectiva del alumnado. Educación XX1, 9, 183-198. doi: https://doi.org/10.5944/educxx1.9.0.325

Mas, O. (2011). El profesor universitario: sus competencias y su formación. Profesorado: Revista de curriculum y formación del profesorado, 15(3), 195-211. Recuperado de http: //www.ugr.es/local/recfpro/rev153COL1.pdf

McAlexander, J. H., Koenig, H. F., \& Schouten, J. W. (2006). Building relationships of brand community in higher education: a strategic framework for university advancement. International Journal of Educational Advancement, 6(2), 107-118.

Mircea, M., \& Andreescu, A. I. (2011). Using cloud computing in higher education: A strategy to improve agility in the current financial crisis. Communications of the IBIMA, 2011, 1-15. doi: https://doi.org/10.5171/2011.875547

Mourad, M., Ennew, C., \& Kortam, W. (2011). Brand equity in higher education. Marketing Intelligence \& Planning, 29(4), 403-420.

Nguyen, N., \& LeBlanc, G. (2001). Image and reputation of higher education institutions in students' retention decisions. International Journal of Educational Management, 15(6), 303-311.

Nunnally, J. C., \& Bernstein, I. H. (1994). Psychometric theory. Nueva York: McGraw- Hill. Olins, W. (2008). Wally Olins: the brand handbook. Londres: Thames \& Hudson.

Palazón-Pérez, A., Gómez-Gallego, M., Gómez-Gallego, J. C., Pérez-Cárceles, M. C., \& Gómez-García, J. (2011). Relación entre la aplicación de metodologías docentes activas y el aprendizaje del estudiante universitario. Bordón. Revista de pedagogía, 63(2), 27-40.

Peltier, J. W., Schibrowsky, J. A., \& Drago, W. (2007). The interdependence of the factors influencing the perceived quality of the online learning experience: A causal model. Journal of Marketing Education, 29(2), 140-153.

Pinar, M., Trapp, P., Girard, T., \& Boyt, T. E. (2014). University brand equity: an empirical investigation of its dimensions. International Journal of Educational Management, 28(6), 616-634. doi: https://doi.org/10.1108/IJEM-04-2013-0051

Rauschnabel, P. A., Krey, N., Babin, B. J., \& Ivens, B. S. (2016). Brand management in higher education: the university brand personality scale. Journal of Business Research, 69(8), 3077-3086. doi: https://doi.org/10.1016/j.jbusres.2016.01.023

Robinson, A., \& Long, G. (1987). Marketing further education: Products or people. Natfhe Journal, 13(3), 42-51. 
Rojas-Méndez, J. I., Vasquez-Parraga, A. Z., Kara, A., \& Cerda-Urrutia, A. (2009). Determinants of student loyalty in higher education: A tested relationship approach in Latin America. Latin American Business Review, 10(1), 21-39. doi: https://doi. org/10.1080/10978520903022089

Smith, B. E. (2003). An investigation of the market orientation of colleges and universities in the council of Christian colleges and universities. (Tesis de doctorado). Recuperada de UMI Microform Service (№ 3129598).

Steiger, J. H. (1990). Structural model evaluation and modification: An interval estimation approach. Multivariate behavioral research, 25(2), 173-180.

Sultan, P., \& Yin Wong, H. (2012). Service quality in a higher education context: an integrated model. Asia Pacific Journal of Marketing and Logistics, 24(5), 755-784. doi: https://doi.org/10.1108/13555851211278196

Thorsteinsson, G., Page, T., \& Niculescu, A. (2010). Using Virtual Reality for Developing Design Communication. Studies in Informatics and Control, 19(1), 93-106.

Wansink, B. (2003). Using laddering to understand and leverage a brand's equity. Qualitative Market Research: An International Journal, 6(2), 111-118.

Waeraas, A., \& Solbakk, M. (2009). Defining the essence of a university: lessons from higher education branding. Higher Education, 57(4), 449-462.

Waterman, A. S. (2014). Service-learning: Applications from the research. Londres: Routledge. Weaver, T. (1976). What is the good of higher education?. Higher Education Review, 8(3), 3-14.

Webb, D., \& Jagun, A. (1997). Customer care, customer satisfaction, value, loyalty and complaining behavior: validation in a UK university setting. Journal of Consumer Satisfaction, Dissatisfaction and Complaining Behavior, 10, 139-151.

Webster, R. L., Hammond, K. L., \& Harmon, H. A. (2006). A study of market orientation in American business schools. Academy of Marketing Studies Journal,9(2), 21-42.

Whisman, R. (2009). Internal branding: a university's most valuable intangible asset. Journal of Product \& Brand Management, 18(5), 367-370. doi: https://doi. org/10.1108/10610420910981846

Wilkie, W. L. (1994). Consumer Behavior. Nueva York: John Wiley \& Sons.

Williams, R. L., \& Omar, M. (septiembre, 2009). Renaming Service Organizations for Growth. Trabajo presentado en Academy of Marketing 5th International Colloquium: Brand, Identity and Corporate Reputation, Cambridge.

Williams, R. L., Williams, H. A., \& Omar, M. (2013). The Marketing Impact of the Principles of Renaming Within a Higher Education Service Organization. Trabajo presentado en American Marketing Association.

Yoo, B., \& Donthu, N. (2001). Developing and validating a multidimensional consumerbased brand equity scale. Journal of business research, 52(1), 1-14.

Zabalza, M. Á. (2009). Ser profesor universitario hoy. La cuestión universitaria, 5, 69-81.

Fecha de recepción: 8 de abril de 2017 .

Fecha de revisión: 19 de abril de 2017.

Fecha de aceptación: 5 de marzo de 2018. 
